\title{
APPLICATION OF DETAILED PHASE COMPARISON PROTECTION MODELS FOR THE ANALYSIS OF ITS OPERATION IN NETWORKS WITH FACTS DEVICES
}

\author{
Nikolay Yu. Ruban ${ }^{1 a}$, Alexandr S. Gusev ${ }^{1}$ and Alexey Yu. Pishchulin ${ }^{1}$ \\ ${ }^{1}$ National Research Tomsk Polytechnic University, 634050 Tomsk, Russia
}

\begin{abstract}
The problem of relay protection misoperations in networks with FACTS devices is considered in the paper. It is offered a solution to this problem for a phase comparison protection of transmission power line through the use of its detailed model for the analysis of the functioning for a case of various normal, emergency and post-emergency modes of electric power systems. The research results of this approach are given in the paper.
\end{abstract}

\section{Introduction}

The electric power industry of Russia in recent years has a tendency to intellectualization, projects of electric power systems with active-adaptive network and FACTS technologies began to appear [1,2]. At the same time it is known that FACTS devices can affect the operation of relay protection and automatic in the nearby power district. Thus, in [3] the need to change the operating characteristic of the distance protection in a case of Unified power flow controller using in the network is described. The modernized resistance relay in [4] is proposed to use if there is a static synchronous compensator on the power line. In both cases, the complexity of distance relay setting is connected with parameters of FACTS devices that depend on the operating mode thus the zone of the relay action is changed. FACTS devices in addition to the distance protection affect the operation of other protections, reacting to the change of the angle, for example basic protection of $110-220 \mathrm{kV}$ power transmission lines phase comparison high-frequency protection (PCP). In connection with this tasks of determining the value of FACTS influence on the PCP functioning and setting calculation of this protection type for power systems with active-adaptive networks are urgent.

\section{Phase comparison protection simulation tools}

The best way to determine the various FACTS devices effect on the PCP is a detailed simulation of a particular PCP type and analysis of transients in its chains.

To create universal detailed mathematical models of electromechanical and microprocessor PCP it is necessary to perform a number of steps:

- to analyze principal circuits of the specific performance of PCP, to develop its appropriate equivalent circuits and to calculate elements parameters;

a Corresponding author : rubanny@tpu.ru

This is an Open Access article distributed under the terms of the Creative Commons Attribution License 4.0, which permits unrestricted use, distribution, and reproduction in any medium, provided the original work is properly cited. 
- to form differential equations that describe processes in the PCP elements;

- to formalize developed mathematical models of PCP in the form of program codes for subsequent implementation in the specialized program of autonomous using and EPS simulation tools.

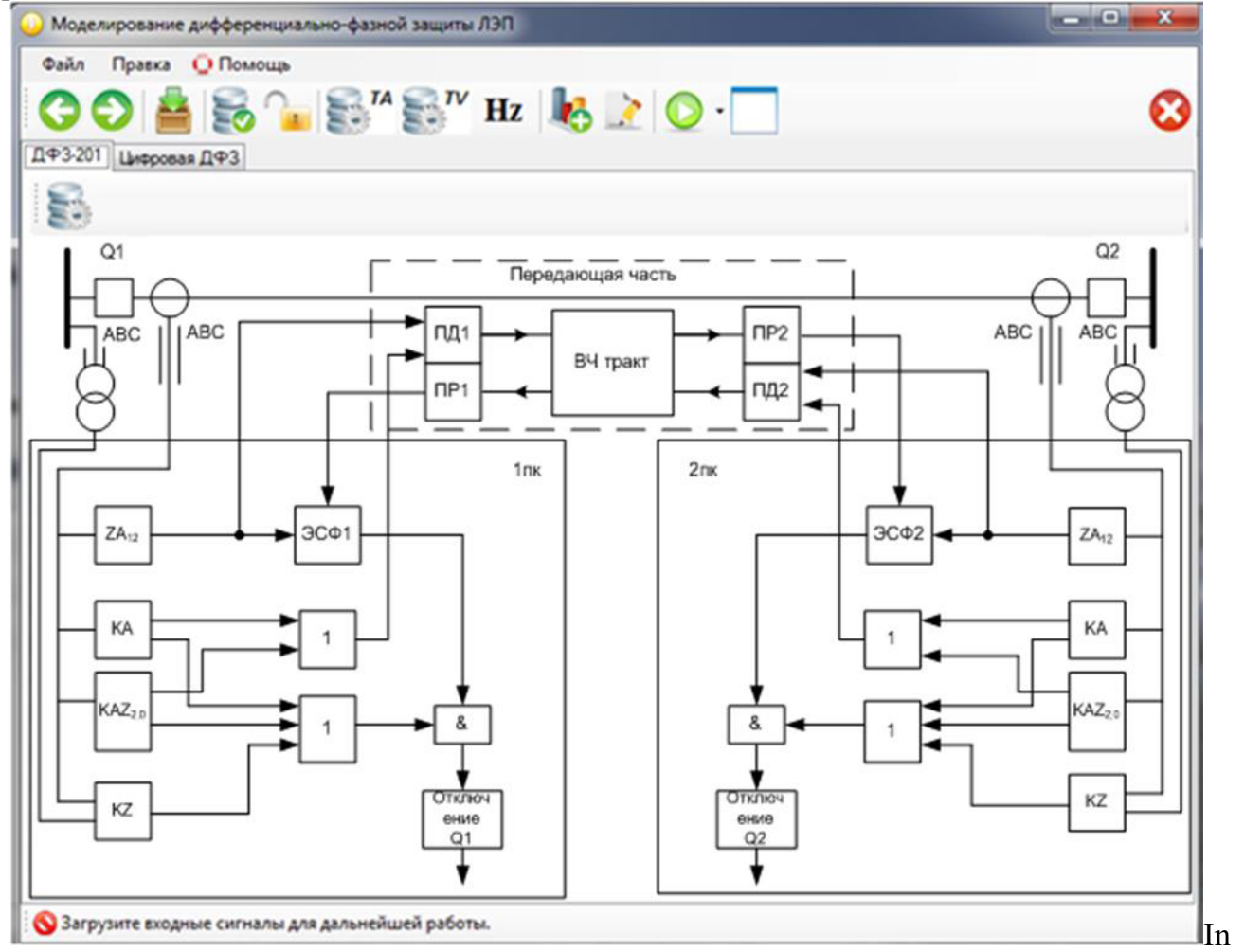

accordance with this methodology the specialized program DPPmodels for PCP simulation has been developed. The main menu view of DPPmodels is shown in Figure 1.

Figure 1. The main menu of DPPmodels.

The DPPmodels program allows to analyze processes in PCP chains on the basis of the recorded primary regime information about emergency in EPS in a Comtrade format. A more detailed description of this program is presented in [5]. However, for a continuous simulation of processes in real time, it is necessary to integrate the program code into the special tool providing adequate modeling of processes in power system [6]. In the role of such tool can serve the Hybrid real time simulator (HRTSim) that has the ability of continuous processes reproduction in the equipment and in EPS as a whole [7]. At the same time the advantage of HRTSim is the lack of integration methodological error component by applying a unique hybrid approach, in which the processes of the equipment are reproduced by analog solutions of differential equations, the relationship between the equipment is carried on the physical level and processes are controled on the digital level. HRTSim external view and structural scheme are given in the figure 2. 

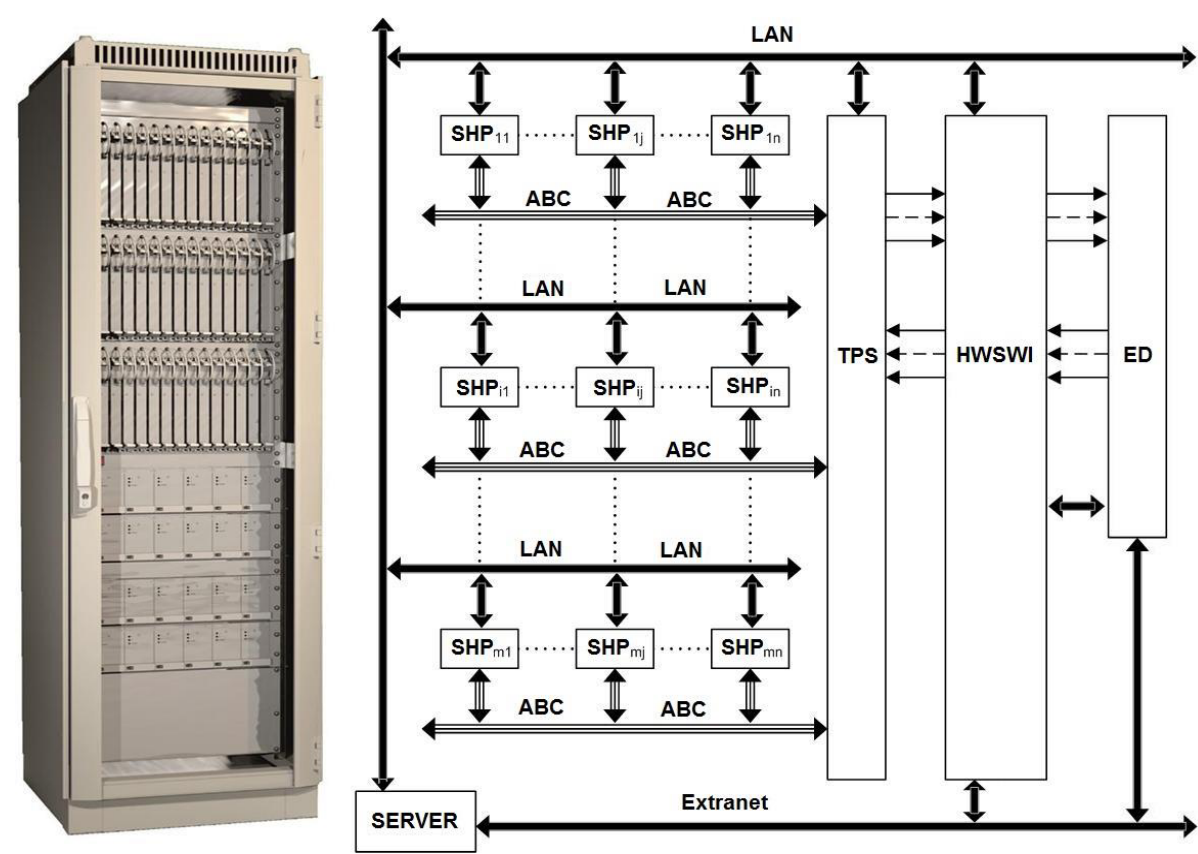

Figure 2. HRTSim external view and structural scheme: SHP - specialized hybrid processors; LAN - local area network; TPS - three-phase switch; HWSWI - hardware/software interface; ED - external device; ABC - threephase input/output of SHP.

Implementation of HRTSim abilities is carried out by developed for this specialized processors (SHP), standardized for all kinds of EPS elements and universal for each of them. The three-phase model physical inputs / outputs of all SHP equipped with digital-controlled series-shunt switches that makes it possible to carry out all kinds of three-phase and per phase switching, including controlled moment of operating. Functional three-phase inputs / outputs of A, B, C phases (ABC in figure 2) of the SHP are interconnected according to the topology of the simulated power system using a threephase switch units (TPS), and their CPU of microprocessor units combined through network switches by local computer network (LAN) with Server, which uses developed for this purpose specialized software. Professional-oriented software of Client work station is created to use HRTSim. There is no limit to set this software on the server or on users' computers in the external computer networks (Extranet), communicating with the server via the Ethernet.

For the interaction with a real equipment (FACTS control stations, relay protection and automation, SCADA systems, a variety of information management system and other external devices (ED)) are developed appropriate software and hardware interfaces (HWSWI) that have matching amplifiers for physical interaction with ED and information linkages to the server and SP for the local and external computer networks.

\section{Experimental results}

At this moment the developed program DPPmodels is investigated for autonomous mode using the primary regime information about the processes in the power system, obtained by HRTSim. As an example, figure 3 shows the waveforms obtained in internal single-phase fault on the protected power line, confirming the difference converted by element of PCP model signal from the idealized signal. 


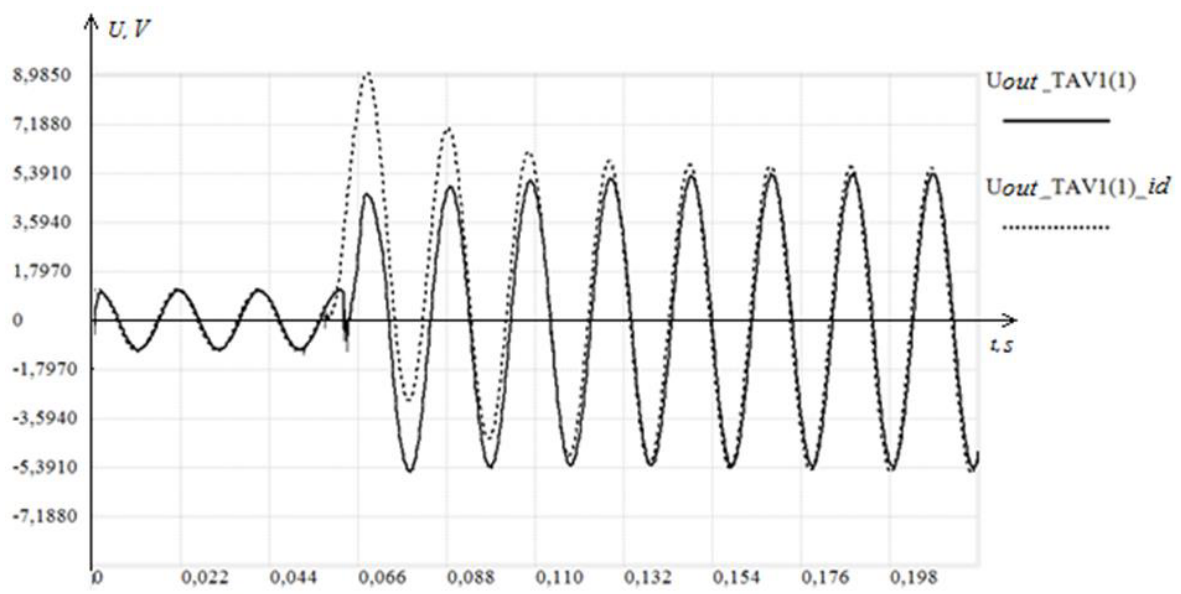

Figure 3. Oscillograms of regime information conversion by intermediate transformer with an air gap of electromechanical PCP

Signal analysis reveals the actual error introduced by the functional units, elements and protection in general into the original information signal. This in turn allows us to estimate the impact of FACTS devices in a network to operation of the particular PCP.

\section{Conclusion}

Integrated into the HRTSim program code of DPPmodels will allow to develop PCP simulation tool reliably and efficiently solving the following important practical problems:

- detailed analysis of the functioning of elements and different versions of PCP as a whole and identifying the causes of malfunction of PCP, including because of the presence of FACTS devices in the network;

- development of measures to eliminate or minimize the identified causes of PCP malfunction;

- adequate setting of various PCP versions for the specific conditions of its operation in EPS., including a closed loop testing of these devices [8].

This work was supported by the Russian Federal Target Program "Research and Development in Priority Fields for the Development of Russian Scientific and Technological Complex for 2014-2020" (Grant agreement № 14.575.21.0104 as of 28.11.2015, the unique identifier of the project RFMEFI57514X0104).

\section{References}

1. P.I. Bartolomey, S.A. Eroshenko, E.M. Lebedev, A.A. Suvorov, Innovative Smart Grid Technologies (ISGT Europe), 2012 3rd IEEE PES International Conference and Exhibition on, 1 (2012).

2. N.I. Voropai, D.N. Efimov, I.N. Kolosok, V.G. Kurbatsky, Smart Grid IEEE Transactions on, 4, $1732(2013)$.

3. S. Jamali, A. Kazemi, H. Shateri, 2009 IEEE Bucharest PowerTech: Innovative Ideas Toward the Electrical Grid of the Future, 1 (2009).

4. S.P. Pandey, M. Tripathy, Proc. IEEE Int. Conf.Power, Control Embedded Syst., 1 (2010).

5. N.Y. Ruban, Y. S. Borovikov, A. O. Sulaymanov, Strategic Technology (IFOST), 2014 9th International Forum on, 264 (2014).

6. E.A. Plesniaev, A.V. Pazderin, Computer as a Tool, 2005. EUROCON 2005.The International Conference on, 2, 1469 (2005) 
7. M. Andreev, A. Sulaymanov, PowerTech, 2015 IEEE, 1 (2015).

8. Yu. Borovikov, A. Prokhorov, M. Andreev, The 7th International Forum on Strategic Technology IFOST2012, 2, 609 (2012). 